\title{
Improvements in Stable Inversion of NARX Models by Using Mann Iteration
}

\author{
J.J.A. Eksteen ${ }^{\mathrm{a} *}$ and P.S. Heyns ${ }^{\mathrm{a}}$ \\ ${ }^{a}$ Department of Mechanical and Aeronautical Engineering, University of Pretoria
}

\begin{abstract}
The use of Mann iteration in the stable inversion of NARX models that have been converted to state space form is investigated to either recover the convergence or improve the accuracy of the best approximate solution under conditions when Picard iteration fails to converge. Attention is given to the use of filtering and time-varying iteration gains. The results are potentially of use in response reconstruction for fatigue testing purposes where the inverse of a NARX model, obtained by system identification, may be used to achieve the reconstruction.
\end{abstract}

Keywords: Stable inversion, Picard iteration, Mann iteration, Ishikawa iteration, iterative learning control, NARX models, discrete time, nonlinear, response reconstruction, fatigue testing.

\section{Introduction}

The exact inversion of square, nonminimum phase nonlinear systems was presented in, amongst others, [1] and [2] for the continuous-time case, and in [3] for the discrete-time case. The procedure is accomplished in three steps, namely (1) the transformation of the nonlinear system to a special form, called the normal form, (2) the inversion of the normal form, and (3) the structuring of the resulting inverse system's state equation as a linear system and partitioning the linear dynamics into stable and unstable parts via an equivalence transformation. This partitioned, linear-like state equation is solved for the bounded solution corresponding to a given desired output of the original nonlinear system via Picard-like iteration. The essential purpose of the iteration is the accommodation of the nonlinear effects of the inverse. Besides being iterative, the calculations are also noncausal and, therefore, must be implemented off-line. The overall procedure is generally referred to as stable inversion, and potentially finds application wherever the inverse of a nonlinear, nonminimum phase system may be used off-line. Examples include achieving exact output tracking in nonlinear, nonminimum phase systems (for example flexible manipulators) by either feed-forward control [1] or iterative learning control (ILC; see [4] for the discrete-time, nonlinear case).

This paper focuses on the situation where the system nonlinearity is of such a degree that Picard iteration fails to converge and stable inversion thus fails to find a solution. This situation is encountered in the paper for a system with polynomial nonlinearity. Generally speaking, to solve the inverse system's state equation via Picard iteration, we effectively convert the first order difference equation to a fixed point problem. It is well known that other iteration schemes besides Picard iteration may also be used to solve the fixed point problem, notably Mann iteration [5] and Ishikawa iteration [6]. While

\footnotetext{
${ }^{*}$ Corresponding author. Email: jan.eksteen@up.ac.za
} 
these iteration schemes are known to display better convergence properties than Picard Iteration in fixed point problems, they have not yet found application in stable inversion for use when Picard iteration fails to converge. This paper investigates the use of Mann iteration and Ishikawa iteration exactly for this purpose in the stable inversion of square, nonlinear discrete-time systems.

The research is part of an investigation into the use of nonlinear, time-domain methods in iterative learning control as used in response reconstruction for fatigue testing purposes (and other purposes such as shock and vibration testing). In such tests the test specimen in the laboratory is typically fitted with electrohydraulic actuators that are under PID feedback control and that mimic the load application to the specimen in normal usage. The goal is to reconstruct in the transducers on the laboratory test specimen desired histories that are representative of normal usage of the structure. The reconstruction is achieved with iterative learning control (ILC), which is an off-line learning control algorithm that in principle calculates the actuator drive signals that reconstructs the desired responses in the specimen transducers. ILC ideally uses an inverse model of the test system in the algorithm. Under the common situation that ILC does not converge over the entire desired test frequency band it is well-known that the more accurate the inverse model, either the wider the frequency band will be over which convergence occurs, or the more accurate the best results obtained prior to the onset of divergence will be. With the actuator drive signals being sent to the PID controllers from a computer and the responses that are measured in the specimen's transducers being saved to the computer disk, it follows that the entire stem between the drive signals and sensors outputs may be modelled as a discrete time system, typically with black box models obtained by system identification. For complex test specimens and sensors on relatively remote locations on the test specimen it follows that the true system dynamics is usually nonlinear. A nonlinear, discrete-time inverse model of the test system for use in ILC may be obtained from nonlinear system identification. The classical and still most common approach is to identify nonlinear ARX models, commonly referred to as NARX models [7]. After having identified the model it needs to be inverted once for every iteration of ILC. This article focuses on how to do this inversion, if not with exact accuracy (due to divergence of stable inversion), then at least as accurately as possible.

The paper is organized as follows: In Section 2 the conversion of a smooth nonlinear system to the normal form is briefly covered and in Section 3 the subsequent formulation of the inverse system is briefly covered. Iterative solution of the inverse system for a given output using different iteration methods is covered in Section 3. Finally, in sections 5 to 7 three examples are presented demonstrating the advantages of Mann iteration over Picard iteration in stable inversion of NARX models, the use of low pass filters to increase accuracy, and the use of different gain schemes in Mann iteration to increase accuracy.

\section{The Normal Form for MIMO Systems}

Consider the square, multiple-input multiple-output (MIMO) nonlinear discrete-time system

$$
\begin{gathered}
x(k+1)=f(x(k), u(k)) \\
y(k)=h(x(k)),
\end{gathered}
$$

with $u(k) \in \Re^{m}, x(k) \in \Re^{n}, y(k) \in \Re^{m}$ and $f$ and $h$ analytic in their domains (and thus smooth). Let $\left(x^{\circ}, u^{\circ}\right)=([0],[0])$ be an equilibrium pair of the system. The output $y_{i}$ has 
local vector relative degree $r_{i}$ around the point $\left(x^{\circ}, u^{\circ}\right)$ if

$$
\frac{\partial h_{i} \circ f_{0}^{l} \circ f(x(k), u(k))}{\partial u_{j}(k)}=0
$$

for $j=1, \ldots, m, i=1, \ldots, m, f_{0}=f(x(k), 0)$, order of iteration $l=0, \ldots, r_{i}-2$, and all $x$ in a neighbourhood of $x^{\circ}$, and there exists a $j_{i} \in\{1, \ldots, m\}$ such that

$$
\left.\frac{\partial h_{i} \circ f_{0}^{r_{i}-1} \circ f(x(k), u(k))}{\partial u_{j_{i}}(k)}\right|_{x^{\circ}, u^{\circ}} \neq 0 .
$$

From Eq. 3 and Eq. 4 the derivative vanishes until the $r_{i}$-th iteration, implying the output is first influenced at time $k+r_{i}$ by the input at time $k$. The vector relative degree is a generalization of the relative degree of single-input single-output (SISO) systems, which for linear time invariant (LTI) systems corresponds to the difference in degree between the denominator and numerator polynomials (in terms of $z$ ). In discrete time systems this difference translates into a delay between the input and the output, with the number of time steps equal to the difference in degree. In practise in laboratory test systems this may be caused by, for example, delay due to the analogue-digital (AD) and digital-analogue (DA) conversion electronics. Define the input-output decoupling matrix as

$$
A(x(k), u(k)):=\left(\frac{\partial y_{i}\left(k+r_{i}\right)}{\partial u_{j}(k)}\right)_{i, j}=\left(\frac{\partial h_{i} \circ f_{0}^{r_{i}-1} \circ f(x(k), u(k))}{\partial u_{j}(k)}\right)_{i, j} .
$$

Assume that

$$
\left.\operatorname{rank} A(x(k), u(k))\right|_{x^{\circ}, u^{\circ}}=m
$$

in which case the system is said to have vector relative degree (or characteristic number) $\left(r_{1}, r_{2}, \ldots, r_{m}\right)$. Assume

$$
0 \in \operatorname{Im}\left(h_{i} \circ f_{0}^{r_{i}-1} \circ f(x(k), u(k))\right) .
$$

In order to derive a coordinate transformation with which to transform the system to the normal form we select the vector

$$
\begin{aligned}
\xi(k) & =\left(\xi^{(1)}(k)^{\mathrm{T}}, \ldots, \xi^{(m)}(k)^{\mathrm{T}}\right)^{\mathrm{T}} \\
\xi^{(i)}(k) & =\left(\xi_{1}^{(i)}(k), \ldots, \xi_{r_{i}}^{(i)}(k)\right)^{\mathrm{T}} \\
& =\left(h_{i}(x(k)), h_{i} \circ f_{0}(x(k)), \ldots, h_{i} \circ f_{0}^{r_{i}-1}(x(k))\right)^{\mathrm{T}} \\
& =\left(y_{i}(k), \ldots, y_{i}\left(k+r_{i}-1\right)\right)^{\mathrm{T}} .
\end{aligned}
$$

The function vector $\xi(k)$ has $|r|:=\sum_{i=1}^{m} r_{i}$ functions. When $|r|<n$, an arbitrary set of $\hat{n}=n-|r|$ additional smooth functions

$$
\eta(k)=\left(\eta_{1}(k), \ldots, \eta_{n-|r|}(k)\right)^{\mathrm{T}}
$$


may always be found such that the function vector

$$
\psi(x(k))=\left(\xi^{\mathrm{T}}(k), \eta^{\mathrm{T}}(k)\right)^{\mathrm{T}}
$$

has a non-singular matrix at $x^{\circ}$, and therefore defines a local coordinate transformation in a neighborhood of $x^{\circ}$ for the system. This coordinate transformation results in the following system in the new coordinates:

$$
\begin{aligned}
\xi_{1}^{(i)}(k+1)= & \xi_{2}^{(i)}(k) \\
\vdots & \\
\xi_{r_{i}-1}^{(i)}(k+1)= & \xi_{r_{i}}^{(i)}(k) \\
\xi_{r_{i}}^{(i)}(k+1)= & \alpha_{i}(\xi(k), \eta(k))+\beta_{i}(\xi(k), \eta(k), u(k)) \\
& i=1, \ldots, m \\
\eta(k+1)= & q(\xi(k), \eta(k), u(k)) \\
y_{1}(k)= & \xi_{1}^{(1)}(k) \\
\vdots & \\
y_{m}(k)= & \xi_{1}^{(m)}(k)
\end{aligned}
$$

This is the normal form for the MIMO system. By setting

$$
\begin{aligned}
\xi_{r_{i}}^{[1, m]} & :=\left(\xi_{r_{1}}^{(1)}, \ldots, \xi_{r_{m}}^{(m)}\right)^{\mathrm{T}} \\
\alpha & :=\left(\alpha_{1}, \ldots, \alpha_{m}\right)^{\mathrm{T}} \\
\beta & :=\left(\beta_{1}, \ldots, \beta_{m}\right)^{\mathrm{T}} \\
\xi_{1}^{[1, m]} & :=\left(\xi_{1}^{(1)}, \ldots, \xi_{1}^{(m)}\right)^{\mathrm{T}}
\end{aligned}
$$

the MIMO normal form may be written more compactly as

$$
\begin{aligned}
\xi_{r_{i}}^{[1, m]}(k+1) & =\alpha(\xi(k), \eta(k))+\beta(\xi(k), \eta(k), u(k)) \\
\eta(k+1) & =q(\xi(k), \eta(k), u(k)) \\
y(k) & =\xi_{1}^{[1, m]}(k)
\end{aligned}
$$

By the definition of vector relative degree (Eq. 6) it follows that $\beta$ (Eq. 19) is a diffeomorphism and may be inverted for the unique $u$, giving the smooth mapping

$$
\begin{aligned}
u(k) & =\gamma\left(\xi(k), \eta(k), \xi_{r_{i}}^{[1, m]}(k+1)\right) \\
& =\beta^{-1}\left(\xi(k), \eta(k), \xi_{r_{i}}^{[1, m]}(k+1)-\alpha(\xi(k), \eta(k))\right) .
\end{aligned}
$$


The non-singular, nonlinear static state feedback $u(k)=\gamma(\xi(k), \eta(k), v(k))$, with $\left(\xi(k)^{\mathrm{T}}, \eta(k)^{\mathrm{T}}\right)^{\mathrm{T}}=\psi(x)$ and $v(k)$ an external control signal, results in

$$
\begin{aligned}
\xi_{1}^{(i)}(k+1)= & \xi_{2}^{(i)}(k) \\
\vdots & \\
\xi_{r_{i}-1}^{(i)}(k+1)= & \xi_{r_{i}}^{(i)}(k) \\
\xi_{r_{i}}^{(i)}(k+1)= & v_{i}(k) \\
& i=1, \ldots, m \\
\eta(k+1)= & q(\xi(k), \eta(k), \gamma(\xi(k), \eta(k), v(k))) \\
:= & \bar{f}(\xi(k), \eta(k), v(k)) \\
y(k)= & \xi_{1}^{[1, m]}(k)
\end{aligned}
$$

Proceeding exactly as in the case of SISO systems we observe that the feedback renders the $\eta(k)$ states unobservable and linearizes the observable part of the system, namely the $\xi$ states. Furthermore, we may define the zero output constrained dynamics as evolutions with $\xi(k)=[0]$ for all $k$ (and thus $v(k)=\xi_{r_{i}}^{[1, m]}(k+1)=[0]$ ). The zero dynamics results in zero output $y(k)=\xi_{1}^{[1, m]}(k)=[0]$, and is characterized by (from Eq. 24)

$$
\eta(k+1)=\bar{f}([0], \eta(k),[0])
$$

The other concepts associated with the zero dynamics, namely the meaning of the zero dynamics and the definition of minimum phase, carry over unchanged from the SISO case.

Note that when the above assumption regarding the input-output decoupling matrix fails, namely when (cf. Eq. 6) $\left.\operatorname{rank} A(x(k), u(k))\right|_{x^{\circ}, u^{\circ}} \neq m$, an algorithm is available for determining the zero dynamics [8], however in which the analysis is not generally local in nature as is the case here.

\section{The Inverse System}

Given a bounded desired output $y_{d}(k), k \in \mathcal{Z}$, the stable inversion problem is to find a desired state trajectory $x_{d}(k)$ and control input $u_{d}(k)$ satisfying Eq. 1, that are in the sequence space $l^{\infty}$ (and thus bounded on $k \in \mathcal{Z}$ ), and that by Eq. 2 results in $y(k)=y_{d}(k)$ for the given system. ( $l^{\infty}$ is a complete, normed vector space; see [9]. A complete, normed vector space is also called a Banach space, and therefore $l^{\infty}$ is a Banach space). In this study $u_{d}$ is also called the desired input. The desired state trajectory and input is found as the bounded solution of the inverse system for the given desired output. We now focus on obtaining the inverse system of the system in the normal form (Eq. 19 and Eq. 20). The output equation of the inverse system is already available as the function $\bar{h}=\gamma, \gamma$ as defined in Eq. 23. With $y(k)=y_{d}(k)$ known, the vectors $\xi(k)$ and $\xi_{r_{i}}^{[1, m]}(k+1)$ follow directly from their definitions. However, to determine $u(k)$ we still need to determine 
$\eta(k)$. To this end we substitute $\bar{h}=\gamma$ into Eq. 20, giving the $\bar{n}$ dimensional system

$$
\begin{aligned}
\eta(k+1) & =q\left(\xi(k), \eta(k), \bar{h}\left(\xi(k), \eta(k), \xi_{r_{i}}^{[1, m]}(k+1)\right)\right) \\
& :=\bar{f}\left(\xi(k), \eta(k), \xi_{r_{i}}^{[1, m]}(k+1)\right) \\
& :=\bar{f}(\eta(k), \Xi(k)),
\end{aligned}
$$

in which $\Xi(k)$ represents $\xi(k)$ and $\xi_{r_{i}}^{[1, m]}(k+1)$. The smoothness $\bar{h}$ and $q$ implies the smoothness of $\bar{f}$. The inverse system is thus given by

$$
\begin{aligned}
\eta(k+1) & =\bar{f}(\eta(k), \Xi(k)) \\
u(k) & =\bar{h}(\eta(k), \Xi(k)) .
\end{aligned}
$$

with $\bar{h}=\gamma$ as in Eq. 23. Now define

$$
\mathcal{U}(\eta(k), \Xi(k)):=\bar{f}(\eta(k), \Xi(k))-A \eta(k)
$$

with

$$
A:=\left.\frac{\partial \bar{f}(\eta(k), \Xi(k))}{\partial \eta(k)}\right|_{[0],[0]}
$$

$A$ has $l$ eigenvalues inside the unit circle and $\hat{n}-l$ eigenvalues outside the unit circle. The state dynamics, Eq. 30, may be restated as

$$
\eta(k+1)=A \eta(k)+\mathcal{U}(\eta(k), \Xi(k)),
$$

which is structured like a linear system - a fact that is subsequently utilized in constructing a bounded solution for the system. Noting the existence of a similarity transformation that transforms $A$ into the Jordan form, in the sequel we assume without loss in generality that Eq. 34 is already in this form, for which

$$
A=\left[\begin{array}{ll}
A_{s} & {[0]} \\
{[0]} & A_{u}
\end{array}\right],
$$

with $A_{s}$ consisting of Jordan blocks representing the $l$ eigenvalues inside the unit circle, and $A_{u}$ consisting of Jordan blocks representing the $\hat{n}-l$ eigenvalues outside the unit circle. The bounded state transition matrix for the linear matrix difference equation $\eta(k+1)=A \eta(k)$ is the $\hat{n} \times \hat{n}$ matrix $\phi(k), k \in \mathcal{Z}$, given as

$$
\phi(k)=\left\{\begin{array}{c}
\left.\left[\begin{array}{c}
A_{s}^{k}[0] \\
{[0]}
\end{array}\right], \quad k>0\right] \\
{\left[\begin{array}{cc}
{[0]} & {[0]} \\
{[0]-A_{u}^{k}}
\end{array}\right], k<0,}
\end{array}\right.
$$


with

$$
\begin{aligned}
& \phi\left(0^{+}\right)=\left[\begin{array}{ll}
I_{s} & {[0]} \\
{[0]} & {[0]}
\end{array}\right] \\
& \phi\left(0^{-}\right)=\left[\begin{array}{ll}
{[0]} & {[0]} \\
{[0]} & -I_{u}
\end{array}\right],
\end{aligned}
$$

and $I_{s}$ and $I_{u}$ the $l \times l$ and $(\hat{n}-l) \times(\hat{n}-l)$ identity matrices respectively. Assuming that $y_{d}(k)$, and thus $\Xi(k), k \in \mathcal{Z}$, is bounded, and that $\bar{f}(\eta(k), \Xi(k))$ is bounded if $\eta(k)$ and $\Xi(k)$ are bounded, it can be shown [3] that the bounded solution $\eta(k)$ of Eq. 34 (and thus Eq. 30) is equivalent to the bounded solution $\eta(k), k \in \mathcal{Z}$, of

$$
\begin{aligned}
\eta(k) & =\sum_{i=-\infty}^{k-1} \phi(k-i) \mathcal{U}(\eta(i-1), \Xi(i-1)) \\
& +\phi\left(0^{+}\right) \mathcal{U}(\eta(k-1), \Xi(k-1)) \\
& +\sum_{i=k+1}^{\infty} \phi(k-i) \mathcal{U}(\eta(i-1), \Xi(i-1)) .
\end{aligned}
$$

For convenience we will designate this solution of Eq. 34 more compactly as

$$
\eta(k)=\sum_{i=-\infty}^{\infty} \phi(k-i) \mathcal{U}(\eta(i-1), \Xi(i-1)) .
$$

Define a non-causal linear operator $G[9]$ to represent the linearly-structured system in Eq. 34 as

$$
\eta=G \mathcal{U}(\eta, \Xi)
$$

The procedure of obtaining the bounded solution, $\eta(k)$, for Eq. 34, and thus Eq. 30, by using the stable solution of $\phi(k)$ in Eq. 37 lies at the heart of stable inversion. Before discussing the calculation of the bounded $\eta(k)$, some norm definitions are in order. Proceeding as in [3], let $\|\cdot\|_{1}$ and $\|\cdot\|_{\infty}$ denote the $l^{1}$ and $l^{\infty}$ norms respectively on $\mathcal{Z}$ [9]. Also let

$$
\|\eta\|_{\infty}=\max _{i}\left\|\eta_{i}\right\|_{\infty}
$$

where $i$ denotes the state vector element.

The function $\mathcal{U}(\eta(k), \Xi(k))$ is said to be uniformly Lipschitz in a closed $s$ neighbourhood of $([0],[0])$ in $(\eta, \Xi)$ space with positive real constants $\left(K_{1}, K_{2}\right)$ if an $s>0$ exists such that for all $\eta_{1}(k), \eta_{2}(k), \Xi_{1}(k)$ and $\Xi_{2}(k)$, all with $\|\cdot\|_{\infty}$ norms $\leq s$, the following local Lipschitz condition holds uniformly $\forall k \in \mathcal{Z}$ :

$\left\|\mathcal{U}\left(\eta_{1}(k), \Xi_{1}(k)\right)-\mathcal{U}\left(\eta_{2}(k), \Xi_{2}(k)\right)\right\|_{\infty} \leq K_{1}\left\|\eta_{1}(k)-\eta_{2}(k)\right\|_{\infty}+K_{2}\left\|\Xi_{1}(k)-\Xi_{2}(k)\right\|_{\infty}$. 
Furthermore, define

$$
\begin{aligned}
\|\phi(k)\|_{\infty} & =\sup \left\{\|\phi(k) c(k)\|_{\infty}:\|c(k)\|_{\infty}=1\right\} \\
\|\phi\|_{1} & =\hat{n} \max _{i, j}\left\|\phi_{i, j}(k)\right\|_{1}
\end{aligned}
$$

where $\phi_{i, j}(k)$ is the $(i, j)$-th element of $\phi(k)$. Finally we note that

$$
\begin{aligned}
\|G \mathcal{U}(\eta, \Xi)\|_{\infty} & =\left\|\sum_{i=-\infty}^{\infty} \phi(k-i) \mathcal{U}(\eta(i-1), \Xi(i-1))\right\|_{\infty} \\
& \leq\|\phi\|_{1}\|\mathcal{U}(\eta, \Xi)\|_{\infty} .
\end{aligned}
$$

\section{Iterative Solution of the Inverse}

\subsection{Introduction}

The bounded $\eta(k)$ satisfying Eq. 37, which is in the form of the solution of a linear system, is iteratively obtained. (The linear system in this case is the nonlinear system Eq. 30, which is a first order nonlinear difference equation, structured as a linear system in Eq. 34.) This is done by recasting Eq. 37 as a fixed point problem by performing iterative searching of the fixed point of Eq. 39. Three of the available iteration schemes that may be used for this purpose are now listed, namely Picard iteration, Mann iteration, and Ishikawa iteration. Consider a mapping $T: B \rightarrow B$, with $B$ a non-empty, convex subset of a normed space $X$. Picard iteration is defined by the sequence $\left(\eta^{(m)}: m \in \mathcal{Z}\right), \eta^{(0)} \in B$, with

$$
\eta^{(m+1)}=T\left(\eta^{(m)}\right)
$$

Mann iteration is defined by the sequence $\left(\eta^{(m)}: m \in \mathcal{Z}\right), \eta^{(0)} \in B, \alpha_{m} \in(0,1]$, with

$$
\eta^{(m+1)}=\left(1-\alpha_{m}\right) \eta^{(m)}+\alpha_{m} T\left(\eta^{(m)}\right)
$$

The usual choice for $\alpha_{m}$ in Mann iteration is $\alpha_{m}=1 /(1+m)$ or $\alpha_{m}=1 / m$.

Finally, Ishikawa iteration is defined by the sequence $\left(\eta^{(m)}: m \in \mathcal{Z}\right), \eta^{(0)} \in B$, $\alpha_{m} \in(0,1], \beta_{m} \in[0,1]$, with

$$
\begin{aligned}
& \eta^{(m+1)}=\left(1-\alpha_{m}\right) \eta^{(m)}+\alpha_{m} T\left(\mu^{(m)}\right) \\
& \mu^{(m)}=\left(1-\beta_{m}\right) \eta^{(m)}+\beta_{m} T\left(\eta^{(m)}\right) .
\end{aligned}
$$

Let $\eta$ designate a fixed point of Picard iteration. Then

$$
\eta=\lim _{m \rightarrow \infty} \eta^{(m)}=\lim _{m \rightarrow \infty} \eta^{(m+1)}
$$

and by taking the limit on both sides of Eq. 46 we get

$$
\eta=\left(1-\alpha_{m}\right) \eta+\alpha_{m} T(\eta)
$$

from which it is clear (after simplification of this equation) that the fixed points of Picard and Mann iteration are equivalent. It can also be shown that a fixed point of 
Picard iteration is also a fixed point of Ishikawa iteration.

Mann and Ishikawa iteration may be simplified by using constant scale factors $\alpha_{m}=\alpha$ and $\beta_{m}=\beta$. Clearly, if $\alpha_{n}=1$ then Picard iteration is obtained from Mann iteration. Furthermore, if $\beta_{m}=0$ then Mann iteration is obtained from Ishikawa iteration.

\subsection{Picard Iteration Method}

The adaptation of Eq. 37 for Picard iteration gives the sequence $\left(\eta^{(m)}(k): m \in \mathcal{Z}\right)$ with

$$
\begin{aligned}
& \eta^{(0)}(k)=[0] \\
& \eta^{(m+1)}(k)=\sum_{i=-\infty}^{\infty} \phi(k-i) \mathcal{U}\left(\eta^{(m)}(i-1), \Xi(i-1)\right)
\end{aligned}
$$

This may be rewritten in operator form as (cf. Eq. 39)

$$
\eta^{(m+1)}=G \mathcal{U}\left(\eta^{(m)}, \Xi\right) .
$$

If the sequence $\left(\eta^{(m)}\right)$ converges then

$$
\eta(k)=\lim _{m \rightarrow \infty} \eta^{(m)}(k)
$$

Sufficient conditions for the convergence of the Picard iteration scheme (Eq. 48) are now presented from [3], with slight modification to the uniqueness aspects, because of its usefulness for subsequent theorems on Mann iteration:

Theorem 4.1: A unique solution $\eta(k) \in l^{\infty}, k \in \mathcal{Z}$, of Eq. 30 exists and is obtained by the Picard iteration of Eq. 48 if:

(1) $\bar{f}$ is uniformly Lipschitz in an $s$ neighbourhood of $([0],[0])$ with Lipschitz constants $\left(K_{1}, K_{2}\right)$

(2) $\|\phi\|_{1} K_{1}<1$,

(3) $\Xi(k) \in l^{\infty}$ with $\|\Xi\|_{\infty} \leq s$, and

(4) $\left(\|\phi\|_{1} K_{2}\|\Xi\|_{\infty} /\left(1-\|\phi\|_{1} K_{1}\right)\right) \leq s$.

Condition 2 implies that Eq. 48 is a contraction ([9] between $\eta^{(m)}$ and $\eta^{(m+1)}$. Condition 4 implies that

$$
\|\phi\|_{1}\left(K_{1} s+K_{2}\|\Xi\|_{\infty}\right) \leq s
$$

This is basically an extension of condition 2 for the additional variable $\Xi$ since, when $\|\Xi\|_{\infty} \rightarrow 0$ this becomes $\|\phi\|_{1} K_{1} \leq 1$, which is satisfied by condition 2 . Theorem 2.1 thus essentially says that if Eq. 37 is a contraction mapping, then Picard iteration converges to the unique fixed point. In the sequel we show that when this is the case Mann iteration also converges. However, when $\|\phi\|_{1} K_{1}>1$, and Eq. 37 is therefore not a contraction, we do not have a general condition of convergence for either Picard, Mann or Ishikawa iteration.

\subsection{Mann Iteration Method}

Consider again a mapping $T: B \rightarrow B$, with $B$ a non-empty, convex subset of a normed space $X$. For the case where $T: B \rightarrow B$ is a contraction, i.e. there exists an $L<1$ such that the Lipschitz condition

$$
\|T x-T y\| \leq L\|x-y\|
$$


is satisfied for all $x, y \in B$, the convergence of Picard, Mann and Ishikawa iteration is proved in Rhoades et al. (2003). However, as in the case above for Eq. 37, when $T$ is $L$ Lipschitz with $L>1$ (and therefore not contractive), we do not have a general condition of convergence of either Picard, Mann or Ishikawa iteration. Convergence conditions for Mann or Ishikawa iteration do exist for some special cases though:

- When $T: B \rightarrow B$ is a nonexpansive with $B$ a non-empty, closed, convex subset of a real Banach space $X$. (An operator $T: B \rightarrow B$ is nonexpansive if $\|T x-T y\| \leq$ $\|x-y\|$ is satisfied for all $x, y \in B$.)

- When $T: X \rightarrow X$ is a uniformly continuous, strongly accretive operator with $X$ an arbitrary real Banach space. (An operator $T: X \rightarrow X$ is strongly accretive if $\|x-y\|<\|x-y+s(T x-T y)\|$ is satisfied for all $x, y \in X$ and $s>0$.)

- When $T: X \rightarrow X$ is $\phi$-strongly accretive and $X$ a real, uniformly smooth Banach space.

- When $T: B \rightarrow B$ is a uniformly continuous, strong pseudocontraction with $B$ a nonempty closed convex bounded subset of an arbitrary real Banach space $X$. (An operator $T: B \rightarrow B$ is a strong pseudocontraction if there exists a $t>1$ such that $\|x-y\| \leq\|(1+t)(x-y)-\operatorname{tr}(T x-T y)\|$ is satisfied for all $x, y \in B$ and $r>0$.)

- When $T: B \rightarrow B$ is a uniformly continuous pseudocontraction with $B$ a nonempty closed convex bounded subset of an arbitrary real Banach space $X$. This is however for a different type of iteration not covered in this study.

The situation that is encountered in most of the theoretical examples in the sequel is that Mann iteration (as well as Picard iteration) diverges for a variety of values of $\alpha_{n}$, suggesting that none of the above-mentioned special cases apply.

The following two theorems are both a result of this research. The first (Theorem 2.2) gives sufficient conditions for the convergence of Mann iteration in stable inversion in rather empirical terms. While of little practical use, it is used to show in the second (Theorem 2.3) that Mann iteration at least converges under weaker conditions than Picard iteration in stable inversion. The theorems are useful in the situation where solution of the inverse system model is not contractive and Picard iteration is likely to diverge, because it implies that at least Mann iteration still has a chance to converge, or can reasonably be expected to give more accurate best results prior to divergence than Picard iteration.

The adaptation of Eq. 38 for Mann iteration gives the sequence $\left(\eta^{(m)}(k): m \in \mathcal{Z}\right)$ with

$$
\begin{array}{ll}
\eta^{(0)}(k) & =[0] \\
\eta^{(m+1)}(k) & =\left(1-\alpha_{m}\right) \eta^{(m)}(k)+\alpha_{m} \sum_{i=-\infty}^{\infty} \phi(k-i) \mathcal{U}\left(\eta^{(m)}(i-1), \Xi(i-1)\right) .
\end{array}
$$

In the sequel we will assume a constant $\alpha_{m}=\alpha$. Eq. 51 may be restated in operator form (using a constant $\alpha$ ) as

$$
\begin{aligned}
\eta^{(m+1)} & =(1-\alpha) \eta^{(m)}+\alpha G \mathcal{U}\left(\eta^{(m)}, \Xi\right) \\
& =(1-\alpha) \eta^{(m)}+\alpha G \mathcal{U}^{(m)}
\end{aligned}
$$

in which we defined $\mathcal{U}^{(m)}:=\mathcal{U}\left(\eta^{(m)}, \Xi\right)$. The adaptation for Ishikawa iteration is done in a similar way. Before deriving convergence conditions some definitions are in order, for 
which we assume $k \in \mathcal{Z}$. As before, define (using Eq. 53)

$$
\begin{aligned}
H^{(m)} & :=\eta^{(m+1)}-\eta^{(m)} \\
& =(1-\alpha)\left(\eta^{(m)}-\eta^{(m-1)}\right)+\alpha G\left(\mathcal{U}^{(m)}-\mathcal{U}^{(m-1)}\right) \\
& =(1-\alpha) H^{(m-1)}+\alpha \tilde{H}^{(m)},
\end{aligned}
$$

in which we defined

$$
\tilde{H}^{(m)}:=G\left(\mathcal{U}^{(m)}-\mathcal{U}^{(m-1)}\right) .
$$

Taking the norm of $\tilde{H}^{(m)}$ we have (using Eq. 44 and Eq. 41):

$$
\begin{aligned}
\left\|\tilde{H}^{(m)}\right\|_{\infty} & =\left\|G\left(\mathcal{U}\left(\eta^{(m)}, \Xi\right)-\mathcal{U}\left(\eta^{(m-1)}, \Xi\right)\right)\right\|_{\infty} \\
& \leq\|\phi\|_{1}\left\|\mathcal{U}\left(\eta^{(m)}, \Xi\right)-\mathcal{U}\left(\eta^{(m-1)}, \Xi\right)\right\|_{\infty} \\
& \leq\|\phi\|_{1} K_{1}\left\|\eta^{(m)}-\eta^{(m-1)}\right\|_{\infty} \\
& =\|\phi\|_{1} K_{1}\left\|H^{(m-1)}\right\|_{\infty} .
\end{aligned}
$$

Define $\tilde{s}_{m} \leq\|\phi\|_{1} K_{1}$ such that

$$
\left\|\tilde{H}^{(m)}\right\|_{\infty}=\tilde{s}_{m}\left\|H^{(m-1)}\right\|_{\infty}
$$

Taking the norm of $H^{(m)}$ (Eq. 55) and defining $F_{m}\left(\alpha, \eta^{(0)}\right) \in[0,1]$ we get

$$
\begin{aligned}
\left\|H^{(m)}\right\|_{\infty} & =F_{m}\left((1-\alpha)\left\|H^{(m-1)}\right\|_{\infty}+\alpha\left\|\tilde{H}^{(m)}\right\|_{\infty}\right) \\
& =F_{m}\left((1-\alpha)\left\|H^{(m-1)}\right\|_{\infty}+\alpha \tilde{s}_{m}\left\|H^{(m-1)}\right\|_{\infty}\right) \\
& =s_{m}\left\|H^{(m-1)}\right\|_{\infty},
\end{aligned}
$$

in which we defined $s_{m}$ as

$$
s_{m}:=F_{m}\left(1-\alpha+\alpha \tilde{s}_{m}\right) .
$$

Theorem 4.2: A solution $\eta(k) \in l^{\infty}, k \in \mathcal{Z}$, of Eq. 30 exists and is obtained by the Mann iteration of Eq. 51 if the following conditions are satisfied:

(1) $\bar{f}$ is uniformly Lipschitz in an $s$ neighbourhood of $([0],[0])$ with Lipschitz constants $\left(K_{1}, K_{2}\right)$,

(2) $\left\|s_{m}\right\|_{\infty}<1$

(3) $\Xi(k) \in l^{\infty}$ with $\|\Xi\|_{\infty} \leq s$, and

(4) $\left(\|\phi\|_{1} K_{2}\|\Xi\|_{\infty} /\left(1-\|\phi\|_{1} K_{1}\right)\right) \leq s$. 
Proof: Let $k \in \mathcal{Z}$. Clearly $\eta^{(0)} \in l^{\infty}$ and $\left\|\eta^{(0)}\right\|_{\infty} \leq s$. Suppose $\eta^{(m)} \in l^{\infty}$ and $\left\|\eta^{(m)}\right\|_{\infty} \leq$ $s$. Taking the norm of Eq. 52 we get (using conditions 1 and 4 ):

$$
\begin{aligned}
\left\|\eta^{(m+1)}\right\|_{\infty} & \leq(1-\alpha)\left\|\eta^{(m)}\right\|_{\infty}+\alpha\left\|G \mathcal{U}\left(\eta^{(m)}, \Xi\right)\right\|_{\infty} \\
& \leq(1-\alpha)\left\|\eta^{(m)}\right\|_{\infty}+\alpha\|\phi\|_{1}\left(K_{1}\left\|\eta^{(m)}\right\|_{\infty}+K_{2}\|\Xi\|_{\infty}\right) \\
& \leq(1-\alpha) s+\alpha\|\phi\|_{1}\left(K_{1} s+K_{2}\|\Xi\|_{\infty}\right) \\
& \leq(1-\alpha) s+\alpha s \\
& =s
\end{aligned}
$$

Thus, by induction $\eta^{(m)} \in l^{\infty}$ and $\left\|\eta^{(m)}\right\|_{\infty} \leq s$ for all $m$. Furthermore, from condition 2 and Eq. 59 it follows that $\left\|H^{(m)}\right\|_{\infty}<\left\|\bar{H}^{(m-1)}\right\|_{\infty}$, and by the ratio test the series $\sum_{m=0}^{\infty}\left\|H^{(m)}(k)\right\|_{\infty}$ is convergent. Hence

$$
\eta^{(m)}(k)=\sum_{j=1}^{m-1} H^{(j)}(k),
$$

$k \in \mathcal{Z}$ is a Cauchy sequence in $l^{\infty}$. Since $l^{\infty}$ is complete ( $l^{\infty}$ is a Banach space), every Cauchy sequence in $l^{\infty}$ converges to an element of $l^{\infty}$, and therefore $\left\{\eta^{(m)}(k)\right\}$ converges (in the $\|\cdot\|_{\infty}$ norm) to an element of $l^{\infty}$. Denote this limit element as $\eta(k), k \in \mathcal{Z}$. Now, for a $\eta_{a}, \eta_{b} \in l^{\infty}$ it follows that

$$
\begin{aligned}
& \left\|(1-\alpha) \eta_{a}+\alpha G \mathcal{U}\left(\eta_{a}, \Xi\right)-\left((1-\alpha) \eta_{b}+\alpha G \mathcal{U}\left(\eta_{b}, \Xi\right)\right)\right\|_{\infty} \\
& =\left\|(1-\alpha)\left(\eta_{a}-\eta_{b}\right)+\alpha G\left(\mathcal{U}\left(\eta_{a}, \Xi\right)-\mathcal{U}\left(\eta_{b}, \Xi\right)\right)\right\|_{\infty} \\
& \leq(1-\alpha)\left\|\left(\eta_{a}-\eta_{b}\right)\right\|_{\infty}+\alpha\left\|G\left(\mathcal{U}\left(\eta_{a}, \Xi\right)-\mathcal{U}\left(\eta_{b}, \Xi\right)\right)\right\|_{\infty} \\
& \leq(1-\alpha)\left\|\eta_{a}-\eta_{b}\right\|_{\infty}+\alpha\|\phi\|_{1} K_{1}\left\|\eta_{a}-\eta_{b}\right\|_{\infty} \\
& =\left(1-\alpha+\alpha\|\phi\|_{1} K_{1}\right)\left\|\eta_{a}-\eta_{b}\right\|_{\infty},
\end{aligned}
$$

implying the uniform continuity of $(1-\alpha) \eta+\alpha G \mathcal{U}(\eta, \Xi)$ with respect to $\eta$, from which

$$
\begin{aligned}
(1-\alpha) \eta+\alpha G \mathcal{U}(\eta, \Xi) & =(1-\alpha) \lim _{m \rightarrow \infty} \eta^{(m)}+\alpha G \mathcal{U}\left(\lim _{m \rightarrow \infty} \eta^{(m)}, \Xi\right) \\
& =\lim _{m \rightarrow \infty}\left((1-\alpha) \eta^{(m)}+\alpha G \mathcal{U}\left(\eta^{(m)}, \Xi\right)\right) \\
& =\lim _{m \rightarrow \infty} \eta^{(m+1)} \\
& =\eta
\end{aligned}
$$

Thus $\eta$ is the fixed point of $(1-\alpha) \eta+\alpha G \mathcal{U}(\eta, \Xi)$ and therefore of Eq. 38, which implies that $\eta(k)$ is the solution of the inverse system's state equation, Eq. 30. $\diamond$

Note that Theorem 2.2 do not prove uniqueness as in Theorem 2.1, mostly because of the restrictive nature of the definition of $F_{m}$. The reason for this choice of $F_{m}$ (and $s_{m}$ ) is to cover all mechanisms contributing to the possible convergence (or less aggressive 
divergence) of Mann iteration compared to Picard iteration when the latter diverges, i.e. when $\|\phi\|_{1} K_{1} \geq 1$ and Eq. 49 is thus not contractive. Substituting $\|\phi\|_{1} K_{1} \geq 1$ into $\tilde{s}_{m}$ in $s_{m}$, we observe that the resulting quantity $S=1-\alpha+\alpha\|\phi\|_{1} K_{1} \geq 1$ is already smaller than $\|\phi\|_{1} K_{1}$, before considering that $\tilde{s}_{m} \leq\|\phi\|_{1} K_{1}$. Further noting that $F_{H}^{(m)} \leq 1$, it is clear that Condition 2, Theorem 4.2 represents a weaker convergence condition than Condition 2, Theorem 4.1. Even if condition 2, Theorem 2.2 fails, Mann iteration may still converge because it is a sufficient condition. If it does not converge, in view of the above considerations it is reasonable to expect better approximate inversion results than with Picard iteration. The following theorem formalizes these issues:

Theorem 4.3: The convergence conditions in Theorem 4.2 for Mann iteration are weaker than the convergence conditions in Theorem 4.1 for Picard iteration since

- if condition 2, Theorem 4.1 is satisfied, then the condition 2, Theorem 4.2 is also satisfied,

- if condition 2, Theorem 4.1 is violated then condition 2, Theorem 4.2 may still be satisfied, and

- if condition 2, Theorem 4.2 is violated, then condition 2, Theorem 4.1 is also violated.

Proof: (1) In this case $\tilde{s}_{m} \leq\|\phi\|_{1} K_{1}<1$. Since $\alpha \in(0,1]$, it follows that $\tilde{s}_{m} \leq 1-\alpha+$ $\alpha \tilde{s}_{m}<1$. Noting that $F_{H}^{(m)} \leq 1$ in Eq. 60 it follows that $s_{m}<1$, and thus $\left\|s_{m}\right\|_{\infty}<1$.

(2) In this case $\tilde{s}_{m}<\|\phi\|_{1} K_{1} \geq 1$. If $\tilde{s}_{m}<1$, then $\tilde{s}_{m} \leq 1-\alpha+\alpha \tilde{s}_{m}<1 \leq\|\phi\|_{1} K_{1}$, and thus $s_{m}<1 \leq\|\phi\|_{1} K_{1}$, implying convergence by Condition 2 . If $1 \leq \tilde{s}_{m} \leq\|\phi\|_{1} K_{1}$, then $1 \leq 1-\alpha+\alpha \tilde{s}_{m} \leq \tilde{s}_{m} \leq\|\phi\|_{1} K_{1}$, and again $s_{m} \leq\|\phi\|_{1} K_{1}$. Therefore $\left\|s_{m}\right\|_{\infty} \leq\|\phi\|_{1} K_{1}$, and condition 2 may still be satisfied, or have less aggressive divergence than Picard iteration.

(3) If $\left\|s_{m}\right\|_{\infty} \geq 1$, then there exists an $m$ such that $s_{m} \geq 1$, implying by Eq. 60 that $1 \leq 1-\alpha+\alpha \tilde{s}_{m} \leq \tilde{s}_{m}$. Recalling that $\tilde{s}_{m} \leq\|\phi\|_{1} K_{1}$, it follows that $\|\phi\|_{1} K_{1} \geq 1 . \diamond$

\subsection{Filter Incorporation}

The results of subsequent theoretical examples show that when stable inversion diverges, incorporating a zero phase low pass filter in the iteration my improve the accuracy of the best results prior to divergence, or even lead to convergence. This is done here by incorporating the filter, which is designated $F$ in operator form, into the inverse system's solution formula, Eq. 39, as follows:

$$
\eta=F G \mathcal{U}(\eta, \Xi)
$$

$F$ is a linear operator representing a zero phase filter based on the causal discrete time filter $F_{0}$ (i.e. in the frequency domain $F$ is represented by $\left|F_{0}\left(e^{j \omega T_{s}}\right)\right|^{2}$ ). We can adapt Eq. 64 for fixed point iteration in the same way as was done for Eq. 39, resulting in the following modified iteration schemes: Picard iteration:

$$
\eta^{(m+1)}=F G \mathcal{U}\left(\eta^{(m)}, \Xi\right)
$$

Mann iteration:

$$
\eta^{(m+1)}=\left(1-\alpha_{m}\right) \eta^{(m)}+\alpha_{m} F G \mathcal{U}\left(\eta^{(m)}, \Xi\right)
$$


Ishikawa iteration:

$$
\begin{array}{r}
\eta^{(m+1)}=\left(1-\alpha_{m}\right) \eta^{(m)}+\alpha_{m} F G \mathcal{U}\left(\mu^{(m)}, \Xi\right) \\
\mu^{(m)}=\left(1-\beta_{m}\right) \eta^{(m)}+\beta_{m} F G \mathcal{U}\left(\eta^{(m)}, \Xi\right)
\end{array}
$$

Note that $F$ above is closely associated with $G$, and therefore impacts the convergence conditions in Theorem 4.1 and Theorem 4.2 in the same way that $G \mathcal{U}$ does. On the practical side, note also that the filter implementation here is of digital low pass filters implemented in the time domain; an alternative frequeny domain implementation in which the FFT frequency band is truncated is in all likelihood also possible, but not tried here.

\section{Example 1: Mann Iteration and Low Pass Filtering - Deterministic Signal}

In this example the advantages of using Mann iteration and low pass filtering is demonstrated for a short-duration deterministic signal. First, however, the ability of both Picard and Mann iteration to converge without using a low-pass filter is demonstrated when the system is operated in a relatively low level of nonlinearity regime.

Consider the following NARX system [10]

$$
\begin{aligned}
y(k)= & \theta_{1} u(k-4)+\theta_{2} u(k-5)+\theta_{3} u(k-6)+\theta_{4} y(k-6) \\
& \theta_{5} u(k-5) y(k-4)+\theta_{6} u(k-5) u(k-6) y(k-4) \\
& +\theta_{7} u(k-5)^{2} u(k-6) y(k-5)
\end{aligned}
$$

with

$$
\left(\theta_{1}, \ldots, \theta_{7}\right)=(0.150,-1 / 12,-1 / 6,1 / 6,-4.0,6.0,11.0)
$$

This kind of nonlinear system may readily be obtained from nonlinear system identification methods [11]. When converted to the nonlinear state space formulation, then to the normal form, and finally inverted, this system gives rise to the following inverse system:

$$
\begin{aligned}
\eta_{1}(k+1) & =C_{1}(k) \eta_{1}(k)+C_{2} \eta_{2}(k)+C_{3}(k) \eta_{1}(k) \eta_{2}(k)+C_{4}(k) \eta_{1}(k)^{2} \eta_{2}(k)+C_{0}(k) \\
\eta_{2}(k+1) & =\eta_{1}(k) \\
u(k-5) & =\eta_{1}(k)
\end{aligned}
$$


$k=1, \ldots, N$, with

$$
\begin{aligned}
C_{0}(k) & =\frac{1}{\theta_{1}} y(k)-\frac{\theta_{4}}{\theta_{1}} y(k-6) \\
C_{1}(k) & =-\frac{\theta_{2}}{\theta_{1}}-\frac{\theta_{5}}{\theta_{1}} y(k-4) \\
C_{2} & =-\frac{\theta_{3}}{\theta_{1}} \\
C_{3}(k) & =-\frac{\theta_{6}}{\theta_{1}} y(k-4) \\
C_{4}(k) & =-\frac{\theta_{7}}{\theta_{1}} y(k-5) .
\end{aligned}
$$

A sample frequency of $250 \mathrm{~Hz}$ is assumed where relevant.

The desired response in question is obtained as the response of Eq. 78 to the following relatively short deterministic signal, $u_{d}(k)$ :

$$
\begin{aligned}
& \bar{u}_{d}(k)= \begin{cases}0, & 1 \leq k \leq 25 \\
c_{u}(\sin (2 \pi(k-31) / 20)+1), & 25<k \leq 46 \\
0, & 46<\leq 146\end{cases} \\
& \tilde{u}_{d}= \bar{F}_{0.16} \bar{u}_{d} \\
& u_{d}= C_{T} \tilde{u}_{d} \\
& F_{0.16}(z)= \frac{0.02287 z^{4}+0.09148 z^{3}+0.13722 z^{2}+0.09148 z+0.02287}{1.00 z^{4}-1.412 z^{3}+1.123 z^{2}-0.40807 z+0.06321} \\
& C_{T}= \begin{cases}0, & 1 \leq k \leq 12 \\
0.5 \sin (2 \pi(k-13) / 24-\pi / 2)+0.5, & 12<k \leq 24 \\
1, & 24<k \leq 122 \\
0.5 \sin (2 \pi(k-123) / 24+\pi / 2)+0.5, & 122<k \leq 134 \\
0, & 134<k \leq 146\end{cases}
\end{aligned}
$$

with $c_{u}=0.165$, the second equation in operator format, and $\bar{F}$ a non-causal linear operator representing the zero phase version of the low pass filter $F(z)$ with cut frequency $40 \mathrm{~Hz}$ (0.16 times the sample frequency). $C_{T}$ as given by Eq. 75 is essentially a sinusoidal taper function. Two cases are performed in this example, namely for $c_{u}=0.25$ (Case 1) and $c_{u}=0.445$ (Case 2). A corresponding desired output trajectory $y_{d}(k)$ was generated for the two $u_{d}(k)$ by applying each $u_{d}(k)$ to the original system (Eq. 68).

We focus on the stable inversion of Eq. 68, i.e. attempting to determine the bounded solution of Eq. 70 by stable inversion for the given $y(k)=y_{d}(k)$ for the two cases. This will be achieved by searching the bounded solution of Eq. 70 by using either Picard or Mann iteration. The desired $u(k)$ may be obtained from the best achieved solution of $\eta$ as $u(k)=\eta_{1}(k+5)$ (cf. Eq. 70). The percentage error between $u_{d}(k)$ and the input 
calculated during iteration $m$ of stable inversion, namely $u^{(m)}(k)$, is defined as:

$$
\begin{aligned}
\operatorname{err}_{1}\left(u^{(m)}\right) & :=100 \frac{\sum_{k=1}^{N}\left|u^{(m)}(k)-u_{d}(k)\right|}{\sum_{k=1}^{N}\left|u_{d}(k)\right|} \\
& =100 \frac{\left\|u^{(m)}(k)-u_{d}(k)\right\|_{1}}{\left\|u_{d}(k)\right\|_{1}} .
\end{aligned}
$$

Similarly

$$
\operatorname{err}_{1}\left(y^{(m)}\right):=100\left\|y^{(m)}(k)-y_{d}(k)\right\|_{1} /\left\|y_{d}(k)\right\|_{1} .
$$

When stable inversion converges towards the desired input signal, the last iteration may be used for the final results. When it diverges, the results of the 'best' needs to be chosen. Since the purpose of stable inversion (and ILC) is the determination of an unknown input signal, the percentage error between $u_{d}(k)$ and the inputs calculated during successive iterations is not normally available. One approach to circumventing this problem is to evaluate some norm of the calculated input signal for successive iterations, and choose an iteration number based on the behavior of this norm over successive iterations. This is referred to here as the input (or $u$ ) - based iteration selection strategy.

Another strategy for selecting the final iteration number is to calculate the output signals that are predicted by the system for the successive, calculated input signals, evaluate the percentage error (or other norm of the error) of each with respect to the desired output signal, and select the iteration number giving the smallest output signal error. This is called here the output (or $y$ ) - based iteration selection strategy, and is the one used in these examples (unless specified otherwise).

The results of stable inversion for the two cases using Picard and Mann iteration is shown in Table 1. For Case 1 both approaches converge even without using a low pass filter in iteration. For Case 2 both approaches diverge when not using a low pass filter in iteration, with Mann iteration faring only slightly worse than Picard iteration for the input, but far better for the output. When using a low pass filter with cut frequency at $100 \mathrm{~Hz}$ in Case 2, Picard iteration still diverges with virtually no improvement from the result obtained without the filter (see Fig. 1). Mann iteration however experiences an oscillatory convergence with best results that are nearly identical to the desired input and output (see Fig. 2). 
Table 1. Example 1: Results of the stable inversion using Picard and Mann iteration. $M$ is the iteration resulting in $\min _{m} \operatorname{err}_{1}\left(u^{(m)}\right)$, i.e. $M=\operatorname{argmin}_{m} \operatorname{err}_{1}\left(u^{(m)}\right)$.

\begin{tabular}{|c|l|c|c|c|c|l|}
\hline Case & $\begin{array}{l}\text { Iteration } \\
\text { type }\end{array}$ & $\alpha_{m}$ & $\begin{array}{c}\min _{m} \operatorname{err}_{1}\left(u^{(m)}\right) \\
{[\%]}\end{array}$ & $\begin{array}{c}\operatorname{err}_{1}\left(y^{(M)}\right) \\
{[\%]}\end{array}$ & $\begin{array}{c}\text { M = Iter. } \\
\text { No. }\end{array}$ & Comment \\
\hline \hline 1 & Picard & 1 & 0.0 & 0.0 & 34 & - \\
\hline 1 & Mann & 0.05 & 0.0 & 0.0 & 33 & - \\
\hline \hline 2 & Picard & 1 & 23.9 & 523 & 1 & - \\
\hline 2 & Mann & 0.2 & 29.3 & 27.7 & 3 & - \\
\hline 2 & Picard & 1.0 & 23.6 & 503 & 31 & $100 \mathrm{~Hz}$ L.P. Filter \\
\hline 2 & Mann & 0.1 & 0.9 & 1.0 & 391 & $100 \mathrm{~Hz}$ L.P. Filter \\
\hline \hline
\end{tabular}




\section{Example 2: Mann Iteration and Low Pass Filtering - Random Signal}

In this example the advantages of using Mann iteration and low pass filtering are demonstrated for a random signal. While the better accuracy of Mann iteration is demonstrated, it is also shown that to achieve convergence, and therefore exact tracking, requires the use of both Mann iteration and a low pass filter in this case.

Consider the following NARX system:

$$
\begin{aligned}
y(k)= & \theta_{1} u(k-4)+\theta_{2} u(k-5)+\theta_{3} u(k-6)+\theta_{4} y(k-4) \\
& \theta_{5} u(k-5) y(k-4)+\theta_{6} u(k-5) u(k-6) y(k-2) \\
& +\theta_{7} u(k-5)^{2} u(k-6) y(k-1)
\end{aligned}
$$

with

$$
\left(\theta_{1}, \ldots, \theta_{7}\right)=(0.150,0.50,0.50,1 / 6,-2.0,6.0,11.0)
$$

A sample frequency of $250 \mathrm{~Hz}$ is assumed. A desired input trajectory, $u_{d}(k)$, is constructed as a random signal with a bandwidth of at most about $50 \mathrm{~Hz}$, and a corresponding desired output trajectory $y_{d}(k)$ is generated by applying $u_{d}(k)$ to the system (Eq. 78). We focus on the stable inversion of this system for the given $y_{d}(k)$. When converted to the nonlinear state space formulation, then to the normal form, and finally inverted, this system gives rise to the following inverse system:

$$
\begin{aligned}
\eta_{1}(k+1) & =C_{1}(k) \eta_{1}(k)+C_{2} \eta_{2}(k)+C_{3}(k) \eta_{1}(k) \eta_{2}(k)+C_{4}(k) \eta_{1}(k)^{2} \eta_{2}(k)+C_{0}(k) \\
\eta_{2}(k+1) & =\eta_{1}(k) \\
u(k-5) & =\eta_{1}(k) \\
k=1, \ldots, N, \text { with } & \\
C_{0}(k) & =\frac{1}{\theta_{1}} y(k)-\frac{\theta_{4}}{\theta_{1}} y(k-4) \\
C_{1}(k) & =-\frac{\theta_{2}}{\theta_{1}}-\frac{\theta_{5}}{\theta_{1}} y(k-4) \\
C_{2} & =-\frac{\theta_{3}}{\theta_{1}} \\
C_{3}(k) & =-\frac{\theta_{6}}{\theta_{1}} y(k-2) \\
C_{4}(k) & =-\frac{\theta_{7}}{\theta_{1}} y(k-1) .
\end{aligned}
$$

The percentage error of the calculated inputs with respect to $u_{d}(k)$ is again calculated as in Example 1 (as well as for the calculated outputs). Stable inversion is now performed for model and desired output as is, and thereafter with a low pass filter incorporated in the iteration. The results of stable inversion using Picard and Mann iteration for the two situations (with and without the filter) is presented in Table 2.

For stable inversion without the low pass filter both iteration schemes are divergent, but with Mann iteration much more accurate than Picard iteration. The iteration error 
for the calculated input is shown in Fig. 4 for the iteration schemes. For stable inversion with a low pass filter with a cut frequency of $50 \mathrm{~Hz}$ Picard iteration is still divergent, but Mann iteration is now convergent. The iteration error for the calculated input is shown in Fig. 5 for the iteration schemes, and the best calculated input in Fig. 6 for Mann iteration showing the impact of the $50 \mathrm{~Hz}$ low pass filter to be virtually imperceptible. 
Table 2. Results of stable inversion. $M$ is the iteration resulting in $\min _{m} \operatorname{err}_{1}\left(u^{(m)}\right)$, i.e. $M=\operatorname{argmin}_{m} \operatorname{err}_{1}\left(u^{(m)}\right)$.

\begin{tabular}{|c|l|c|c|c|c|l|}
\hline Case & $\begin{array}{l}\text { Iteration } \\
\text { type }\end{array}$ & $\alpha_{n}$ & $\begin{array}{c}\min _{m} \operatorname{err}_{1}\left(u^{(m)}\right) \\
{[\%]}\end{array}$ & $\begin{array}{c}\operatorname{err}_{1}\left(y^{(M)}\right) \\
{[\%]}\end{array}$ & $\begin{array}{c}\mathrm{M}=\text { Iter. } \\
\text { no. }\end{array}$ & Comment \\
\hline \hline 1 & Picard & 1.0 & 36.5 & 200.9 & 1 & No filter \\
\hline 1 & Mann & 0.5 & 9.4 & 6.1 & 4 & No filter \\
\hline \hline 2 & Picard & 1.0 & 36.1 & 204.8 & 1 & 50 Hz L.P.F. \\
\hline 2 & Mann & 0.5 & 0.7 & 0.4 & $100+$ & $50 \mathrm{~Hz}$ L.P.F. \\
\hline
\end{tabular}




\section{Example 3: Evaluation of Various Mann Iteration Gain Strategies}

In this example the advantages of using Mann iteration and low pass filtering is demonstrated for a random signal, however, whereas in examples 1 and 2 a constant gain was used in Mann iteration, in this example various strategies for the gain in Mann iteration are evaluated, including iteration-dependent gains, and time-dependent gains. The iteration-dependent gain strategy involves a monotonically decreasing sequence of gain values, while the time-dependent gain strategy is essentially an amplitude dependent formula (intended to interrupt the process of local divergence, which is a frequent occurrence). It is found that the most accurate results are achieved when employing a combined iteration-dependent and time-dependent gain.

In Example 3 we focus on the stable inversion of the same system as in Example 2, namely Eq. 78, however with a larger-amplitude $u_{d}(k)$ and corresponding $y_{d}(k)$ (Fig. 7). In view of the polynomial nature of the system this generally implies the system is now operated in a more nonlinear regime. Once again, the percentage error between signals is calculated as in Example 1. In a subsequent chapter on ILC we will attempt to invert this system for this $y_{d}(k)$ by means of ILC. (Recall that ILC iteratively employs stable inversion.) We will compare the success of inversion of this system via ILC with that of the 'single pass' stable inversion in this example, the latter serving as a base line for the comparison. Three approaches to handling the gain in Mann iteration will be tried, namely constant gains, monotonically decreasing gain sequences, and time-varying gains (either constant or decreasing):

(1) Preliminary trials using constant gains: Noting that if $\alpha_{m}=1$ we recover Picard iteration from Mann iteration and, in turn, if $\beta_{m}=0$ we recover Mann iteration from Ishikawa iteration, it follows that Ishikawa iteration for all combinations of constant $\alpha_{m}=\alpha$ and $\beta_{m}=\beta$ (each varying over regular intervals) includes both Mann iteration (also with constant $\alpha_{m}$ ) and Picard iteration. A mapping for the minimum iteration error for the input signal achieved with Ishikawa iteration for different combinations of $\alpha$ and $\beta$ is shown in Fig. 8. A low pass filter of $50 \mathrm{~Hz}$ was used. The curve $\beta=0$ thus represents Mann iteration, and the point $\alpha=1$ and $\beta=0$ represents Picard iteration. Clearly Ishikawa iteration (evaluated using a constant $\alpha$ and $\beta$ ) does not significantly improve on the best results that may be obtained with Mann iteration (evaluated at the same $\alpha$ ), while Mann iteration does represent a significant improvement on Picard iteration. For the remainder of this study we will therefore focus on Mann iteration only during stable inversion. Noting in Fig. 8 that the accuracy of stable inversion is greater for smaller values of $\alpha$ (and $\beta$ ), in the remainder of the example we therefore focus on smaller values of $\alpha$ in particular. We will also employ a zero phase low pass filter with a suitable cut frequency in stable inversion and set $\eta_{i}^{(0)}(k)=0$ for all $k$ and $i$.

(2) Systematic trials using constant gains: Firstly we evaluate the stable inversion results that are obtained using constant gains over a range of values. For this we use a $50 \mathrm{~Hz}$ zero phase low pass filter, a $y$-based iteration selection strategy (as before), and limit the number of iterations to at most 10000. The whole exercise is repeated for a $70 \mathrm{~Hz}$ and $90 \mathrm{~Hz}$ filter, and for no filter. The best results are summarized in Table 3 (constant gain, time-independent case). The most accurate calculated $u$ signal has an error of $27.6 \%$, and the corresponding output signal an error of $26.0 \%$.

(3) Using monotonically decreasing gain sequences: Next we evaluate the stable inversion results that are obtained using monotonically decreasing gains $\left(\alpha_{n}\right)$. [12] 
suggest the following prototype formula for $\alpha_{n}, n \geq 0$ :

$$
\alpha_{n}=\frac{1}{1+n},
$$

which we generalize here as follows:

$$
\alpha_{n}=n_{0} \frac{\alpha_{0}-\alpha_{\lim }}{(n+1)^{\nu}+\left(n_{0}-1\right)}+\alpha_{\lim },
$$

$n \geq 0$ and $\alpha_{0}$ the initial value of $\alpha_{n}$ (i.e. at $n=0$ ). Using a $50 \mathrm{~Hz}$ zero phase low pass filter a rough optimization exercise of the stable inversion results for $\alpha_{\text {lim }}$, $n_{0}$ and $\nu$ indicates best results are obtained for $\alpha_{\text {lim }}$ between 0.0001 and 0.0005 , $n_{0}=2$ and $\nu=1$. In the sequel therefore we will use

$$
\alpha_{n}=2 \frac{\alpha_{0}-\alpha_{\lim }}{n+2}+\alpha_{\lim },
$$

$n \geq 0$. Using a $50 \mathrm{~Hz}$ zero phase low pass filter, a $y$-based iteration selection strategy, at most 10000 iterations, and various combinations of $\alpha_{0}$ and $\alpha_{\mathrm{lim}}$, with

$$
\alpha_{0} \in\{-1.0,-0.7,-0.5,-0.3,-0.1,-0.05\}
$$

and

$$
\alpha_{\lim } \in\{0.1,0.05,0.01,0.005,0.001,0.0005,0.0001\}
$$

gives best stable inversion results as in Table 3 (decreasing gain, time-independent case). Note that the most accurate calculated $u$ signal is $16.8 \%$, and when simulated back through the system gives an output error of $12.4 \%$. Clearly the use of a decreasing gain sequence gives more accurate stable inversion results, but at the cost of larger numbers of required iterations, and therefore takes longer. Results obtained using a $70 \mathrm{~Hz}, 90 \mathrm{~Hz}$ and absent zero phase low pass filter are also shown in the table.

(4) Time-varying gains: The above procedure may be repeated using a time-varying gain with the aim of suppressing the localised radical signal growth over successive iterations that often result in divergence of stable inversion. Various approaches exist for designing such a gain function that is dependent on the local magnitude of a signal for a given iteration as a function of time and on the nominal value of $\alpha_{n}$. These approaches tend to be highly empirical in nature and are therefore not further elaborated on here. The best results obtained with this formula are presented in Table 3 (for both a constant nominal $\alpha_{n}$ and decreasing nominal $\alpha_{n}$, the latter once again obtained for various combinations of filter frequency, $\alpha_{0}$ and $\alpha_{\mathrm{lim}}$ ). The best results obtained with iteration-independent, time-varying gains represent an improvement over the results of Case 2 and Case 3 . The best results obtained with decreasing, time-varying gains represent the best stable inversion results thus far for this system and the given desired output signal. These results demonstrate the advantage of allowing $\alpha_{n}$ to be time-varying, for both both iteration-independent and iteration-dependent nominal values of $\alpha_{n}$. 
Table 3. Best results of stable inversion of Eq. 78. $u_{e r r}=\min _{m} \operatorname{err}_{1}\left(u^{(m)}\right) \cdot y_{e r r}=\operatorname{err}_{1}\left(u^{(M)}\right)$, where $M$ is the iteration resulting in $\min _{m} \operatorname{err}_{1}\left(u^{(m)}\right)$, i.e. $M=\operatorname{argmin}_{m} \operatorname{err}_{1}\left(u^{(m)}\right)$.

\begin{tabular}{|l|c|c|c|c|l|}
\hline Iteration type & $\begin{array}{c}\text { Filter cut } \\
\alpha_{n}\end{array}$ & $\begin{array}{c}u_{\text {err }} \\
\text { freq. [Hz] }\end{array}$ & $\begin{array}{c}y_{\text {err }} \\
{[\%]}\end{array}$ & $\begin{array}{c}\text { M = Iter. } \\
\text { no. }\end{array}$ & Comment \\
\hline Iteration-independent & 50 & 27.6 & 26.0 & 13 & $\alpha_{n}=0.1$ \\
Time-independent & 70 & 33.4 & 31.7 & 913 & $\alpha_{n}=0.001$ \\
& 90 & 37.7 & 33.4 & 808 & $\alpha_{n}=0.001$ \\
& (None) & 66.0 & 55.0 & 492 & $\alpha_{n}=0.001$ \\
\hline Iteration-dependent & 50 & 16.8 & 12.4 & 10000 & $\alpha_{0}=0.05, \alpha_{\lim }=0.0001$ \\
Time-independent & 70 & 29.7 & 25.4 & 3697 & $\alpha_{0}=0.05, \alpha_{\lim }=0.0001$ \\
& 90 & 35.6 & 27.9 & 3160 & $\alpha_{0}=0.05, \alpha_{\lim }=0.0001$ \\
& (None) & 65.7 & 51.7 & 234 & $\alpha_{0}=0.05, \alpha_{\lim }=0.0001$ \\
\hline Iteration-independent & 50 & 15.7 & 10.3 & 3776 & $\alpha_{n}=0.001$ \\
Time-varying & 70 & 19.5 & 14.5 & 12 & $\alpha_{n}=0.5$ \\
& 90 & 26.2 & 16.1 & 423 & $\alpha_{n}=0.01$ \\
& (None) & 46.4 & 46.6 & 3 & $\alpha_{n}=0.3$ \\
\hline Iteration-dependent & 50 & 13.3 & 8.1 & 8327 & $\alpha_{0}=0.05, \alpha_{\lim }=0.0005$ \\
Time-varying & 70 & 18.8 & 15.1 & 85 & $\alpha_{0}=0.5, \alpha_{\lim }=0.005$ \\
& 90 & 28.4 & 13.5 & 3244 & $\alpha_{0}=0.3, \alpha_{\lim }=0.0001$ \\
& (None) & 48.6 & 38.6 & 18 & $\alpha_{0}=0.3, \alpha_{\lim }=0.0005$ \\
\hline
\end{tabular}




\section{Discussion}

The paper outlines a method for the inversion of nonlinear systems, with the immediate application that is presented here being the inversion of nonlinear models obtained by system identification for use in ILC in laboratory test systems. This capability can potentially find extensive use in response reconstruction for purposes of road simulator fatigue tests (with the desired response trajectory a field measured response) or shock and vibration tests (using a synthetic desired response).

For routine application in this way the methods presented here need to be implemented systematically and generically, which is very possible and has indeed been done by the first author in a Matlab based software package (used for both the the numeric work and the graphic user interface) utilizing National Instruments AD and DA hardware. To this end the nonlinear system can be limited to an input-affine (or control-affine) NARX system, which can readily be identified using non-iterative least squares, and (if needed) subsequently converted to state space form. In fact, completing the process to obtain the inverse state space model reveals convenient connections and similarities to the original NARX model, ultimately enabling the intermediate state space step to be skipped in practice.

The disadvantage to limiting the nonlinear system to NARX models, and indeed to using the stable inversion method in the first place, is that it is limited to smooth nonlinear models (as NARX models are). That doesn't imply the method is not useful in more general systems featuring non-smooth nonlinearity, because such systems can indeed be approximated with smooth systems, allowing ILC to compensate for the model deficiencies via its learning capacity (which is well known to be able to account for a certain level of system nonlinearity when, for example, using a linear model).

Recommendations for further research on stable inversion:

- The time dependent gain employed in Mann iteration in stable inversion was found to be of value. There are many possibilities for the design of the time-dependence function, and the best approach need to be found.

- This research didn't devote much attention t'o the use of Ishikawa iteration in stable inversion. Further work is needed to investigate the possible utility of Ishikawa iteration in improving the results of stable inversion of NARX models, including the use of iteration dependent and time dependent gains.

- Theorem 4.3 for showing that Mann iteration converges under weaker conditions than Picard iteration may be improved with further research, possibly leading to or requiring changes to Theorem 4.2.

- This paper omits the details of conversion of an identified NARX model to the corresponding state space form (details may be found in [14]) on the grounds that it is routine, however there is scope for tailoring the analysis presented here and extending it for the NARX-derived state space formulation specifically.

\section{Conclusions}

The use of Mann iteration and a low pass filter was introduced for stable inversion. In the examples for stable inversion of NARX models Mann iteration clearly gave better results than Picard iteration, with best results achieved when the Mann iteration gains were both iteration dependent and time varying. The value of using a low pass filter in stable inversion was also clearly showed in the examples. 


\section{Funding}

This work was supported by the National Research Foundation and Investmech, Pty. Ltd.

\section{References}

[1] Devasia S, Chen D, Paden B. Nonlinear inversion-based output tracking. IEEE Trans. on Automatic Control. 1996;AC-41:(7):930-942.

[2] Hunt LR, Meyer G. Stable inversion for nonlinear systems. Automatica. 1997;33(8):15491554.

[3] Zeng G, Hunt LR. Stable inversion for nonlinear discrete-time systems. IEEE Trans. on Automatic Control. 2000;45(6):1216-1220.

[4] Markusson O. Model and system inversion with applications in nonlinear system identification and control, Doctoral thesis, Royal Institute of Technology, Stockholm, Sweden; 2002.

[5] Mann WR. Mean value in iteration. Proc. of the Am. Math. Soc. 1953;4:506-510.

[6] Ishikawa S. Fixed points by a new iteration method. Proc. of the Am. Math. Soc. 1974;44:147150 .

[7] Billings, SA. Nonlinear system identification, John Wiley and Sons; 2013.

[8] Monaco S, Normand-Cyrot D. Minimum-phase nonlinear discrete-time systems and feedback stabilization. Proc. of the 26th IEEE Conf. on Decision and Control. 1987;979-986.

[9] Kreyszig E. Introductory functional analysis with aplications. John Wiley and Sons; 1978.

[10] Chen S, Billings SA. Representations of non-linear systems: the NARMAX model. Int. Journal of Control. 1989;49(3):1013-1032.

[11] Chen S, Billings SA. Orthogonal least squares methods and their application to non-linear system identification. Int. Journal of Control. 1989;50(5):1873-1896.

[12] Chidume CE, Osilike MO. Iterative solutions of nonlinear accretive operator equations in arbitrary banach spaces. Nonlinear Analysis. 1999;36:863-872.

[13] Osilike MO. Iterative solutions of nonlinear equations of the accretive type. Nonlinear Analysis. 2000;42:291-300.

[14] Eksteen JJA. Advances in iterative learning control with application to structural dynamic response reconstruction [unpublished doctoral dissertation]. Pretoria (South Africa): Universiy of Pretoria; 2014. 


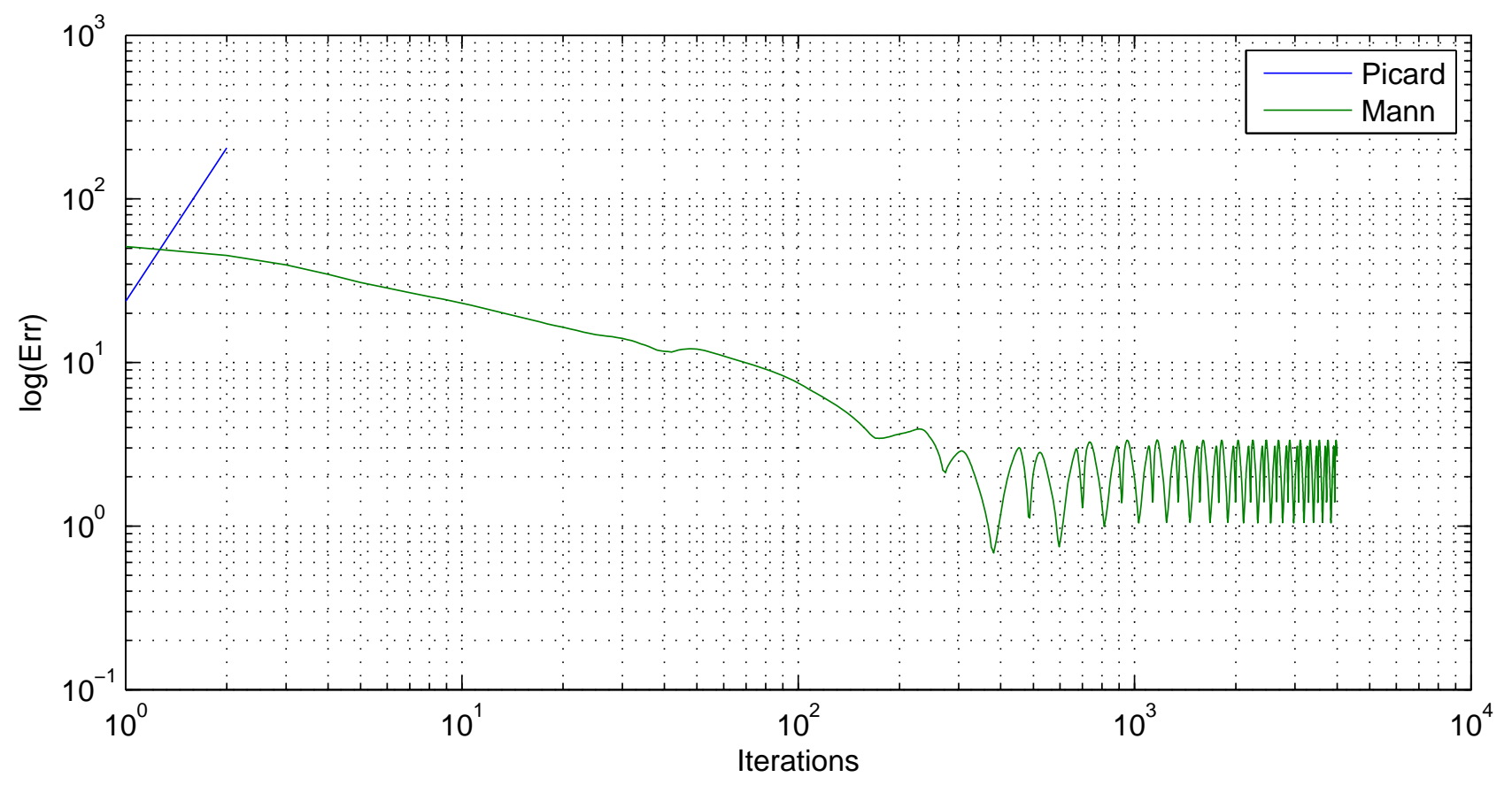

Figure 1. Ex. 1, Case 2: Iteration error for calculated input signal for Picard and Mann iteration.

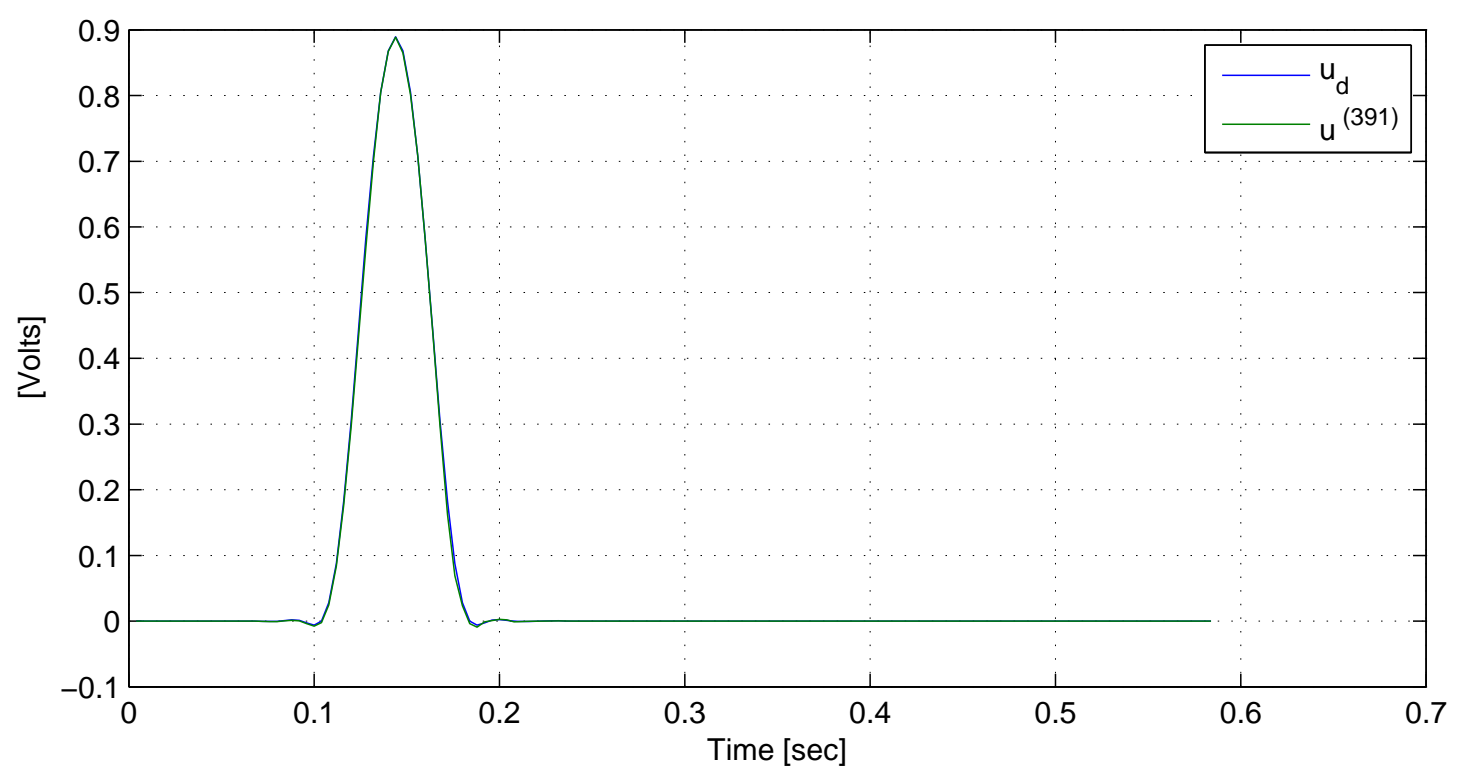

Figure 2. Ex. 1, Case 2: Best $u^{(i)}(t)$ for Mann iteration. 


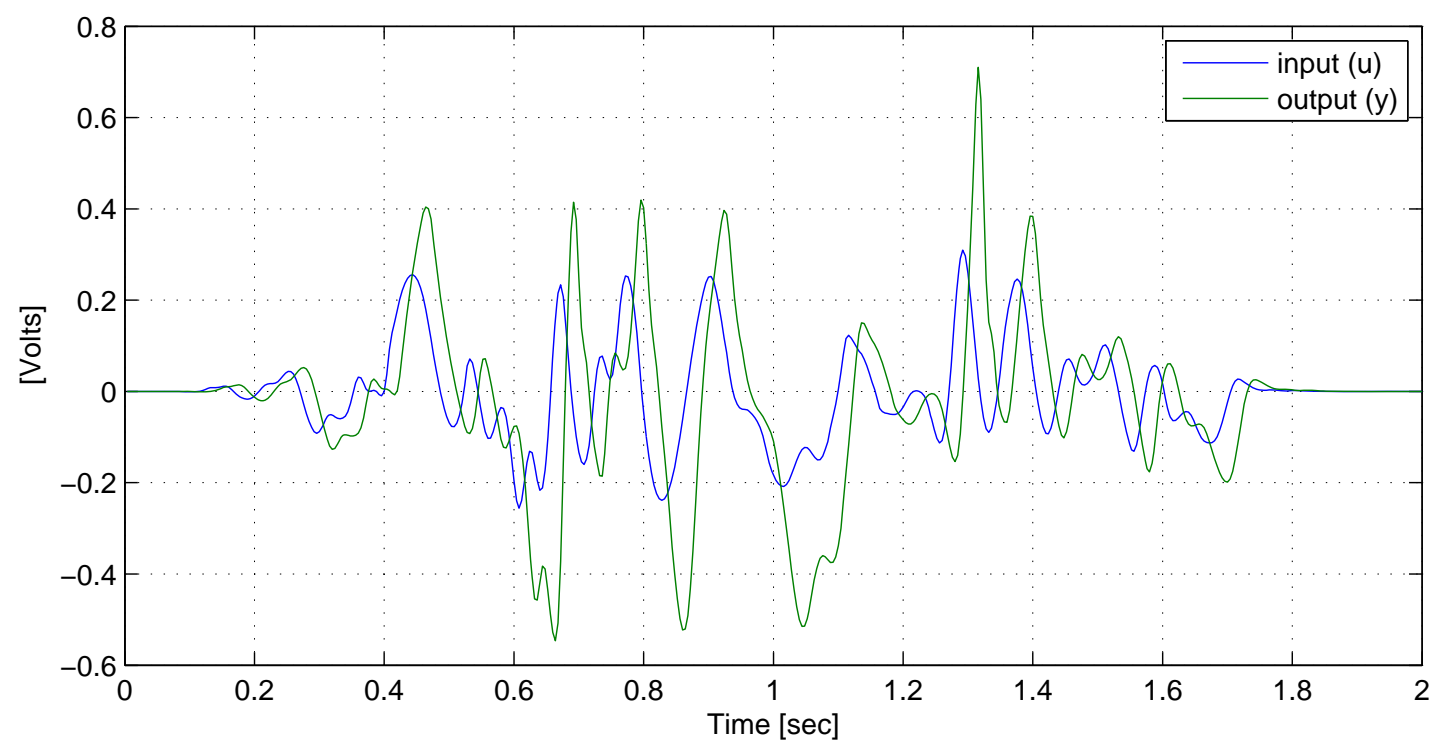

Figure 3. Exp. 2: $u_{d}(t)$ and $y_{d}(t)$.

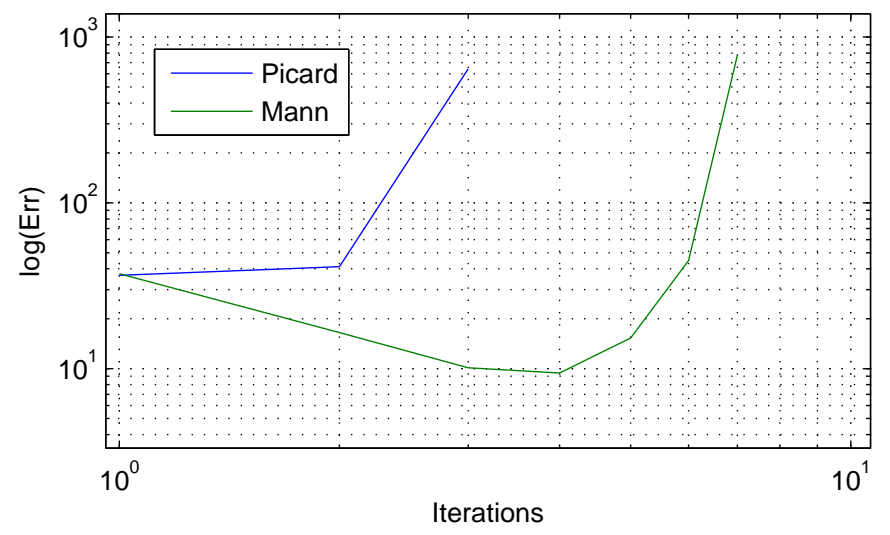

Figure 4. Exp. 2 (no low pass filter): Iteration error of calculated inputs for Picard and Mann iteration. 


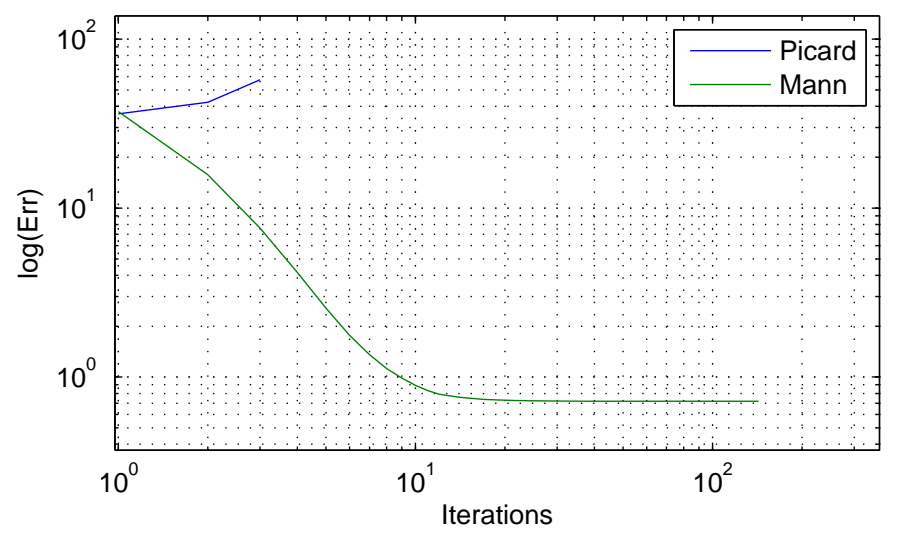

Figure 5. Exp. $2(50 \mathrm{~Hz}$ low pass filter): Iteration error of calculated inputs for Picard and Mann iteration.

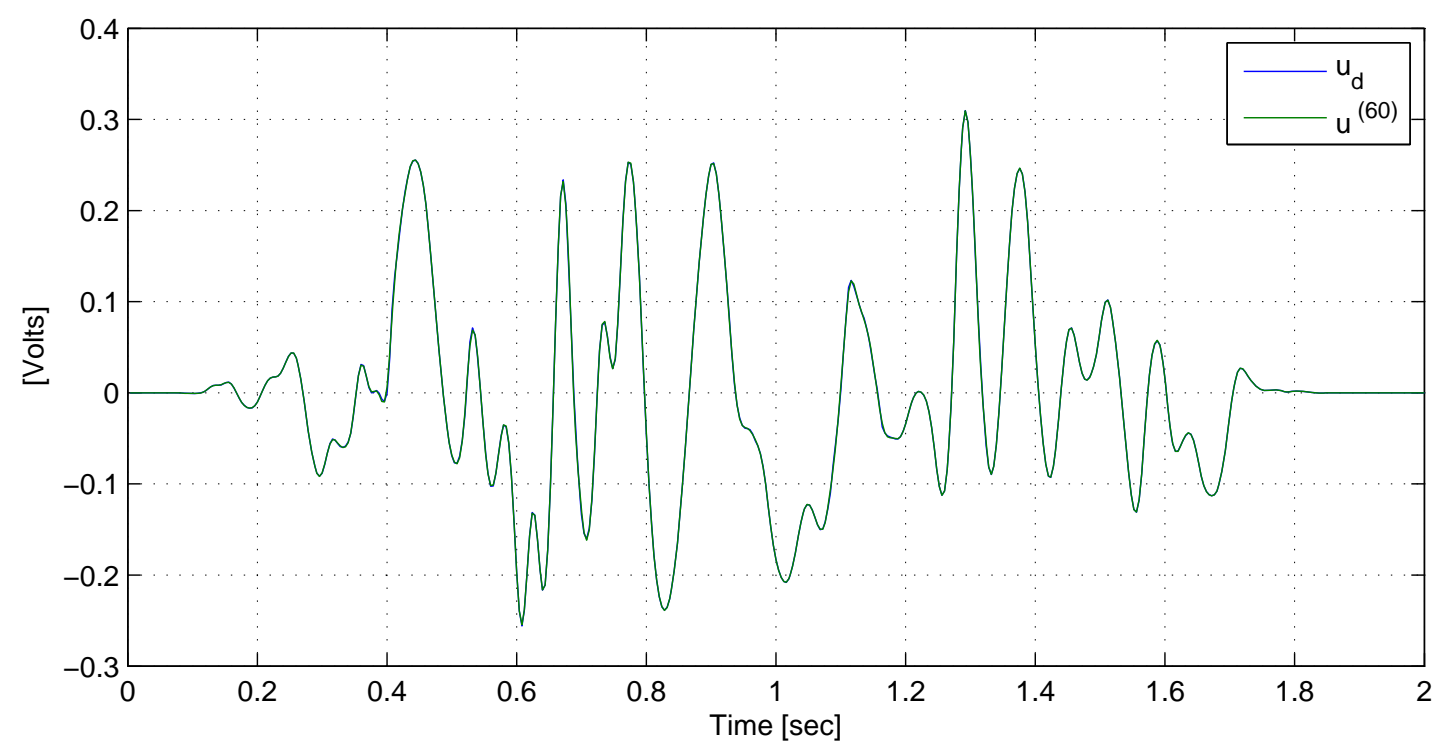

Figure 6. Exp. $2\left(50 \mathrm{~Hz}\right.$ low pass filter): Best $u^{(i)}$ for Mann iteration. 

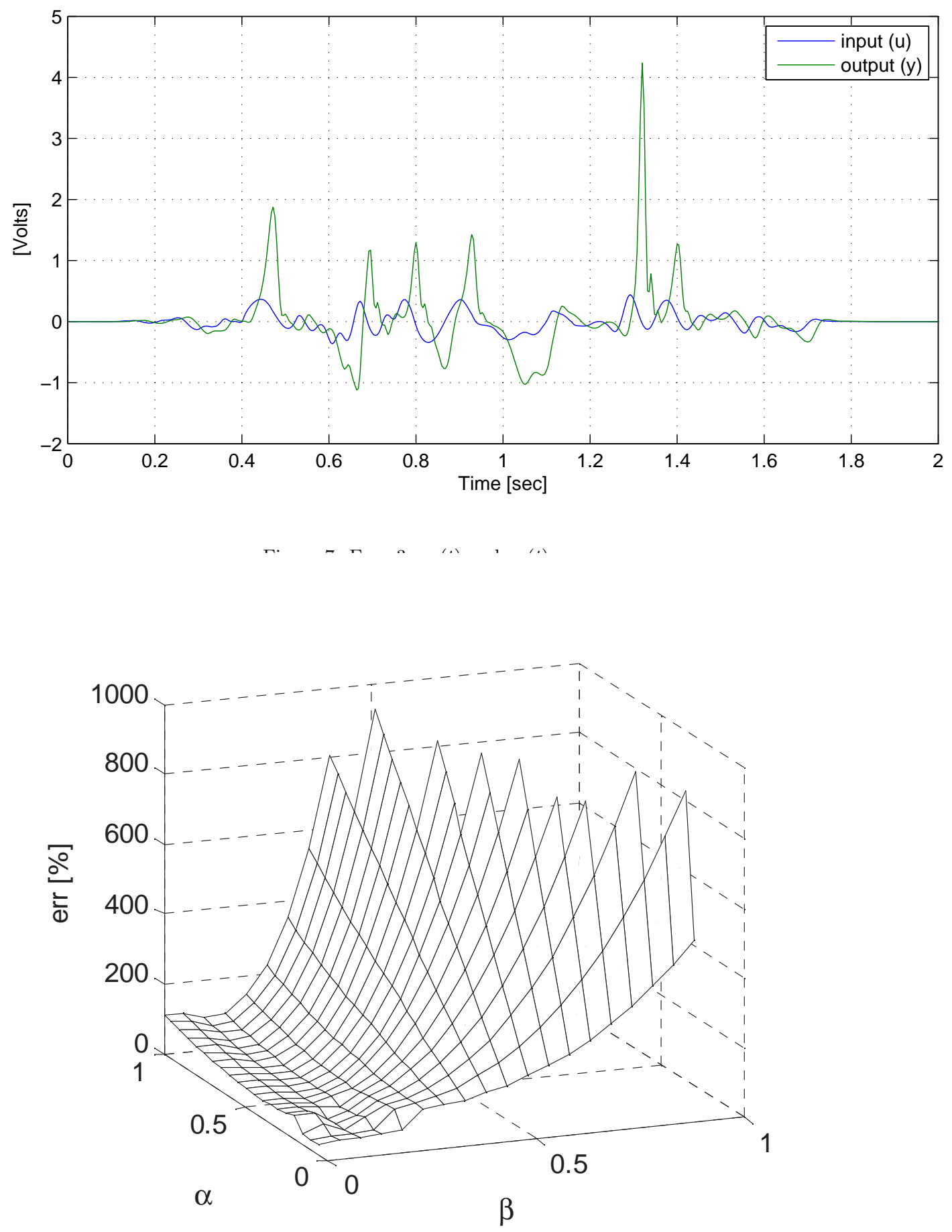

Figure 8. Minimum iteration error for the input using a constant $\alpha$ and $\beta$, for Ishikawa iteration. 